Bull. Austral. Math. Soc.

$34 \mathrm{M} 05,30 \mathrm{D} 35$

VOL. 66 (2002) [331-343]

\title{
ON MEROMORPHIC SOLUTIONS OF CERTAIN NONLINEAR DIFFERENTIAL EQUATIONS
}

\author{
J. Heittokangas, R. Korhonen AND I. LAine
}

In this paper, we consider the growth of meromorphic solutions of nonlinear differential equations of the form $L(f)+P(z, f)=h(z)$, where $L(f)$ denotes a linear differential polynomial in $f, P(z, f)$ is a polynomial in $f$, both with small meromorphic coefficients, and $h(z)$ is a meromorphic function. Specialising to $L(f)-p(z) f^{n}=h(z)$, where $p(z)$ is a small meromorphic function, we consider the uniqueness of meromorphic solutions with few poles only. Our results complement earlier ones due to C.-C. Yang.

\section{INTRODUCTION}

In a recent paper [7], C.-C. Yang considered transcendental entire solutions $f$ of finite order of

$$
L(f)-p(z) f^{n}=h(z)
$$

where $L(f)$ denotes a linear differential polynomial in $f$ with polynomial coefficients, $p(z)$ is a non-vanishing polynomial, $h(z)$ is entire and $n \geqslant 3$. In particular, he showed that $f$ has to be unique, unless $L(f) \equiv 0$.

In this paper, we consider a slightly more general form of the equation (1.1), where $p(z), h(z)$ and the coefficients of $L(f)$ are meromorphic, and not necessarily of finite order. We show that the method used by Yang can be modified to obtain similar uniqueness results for meromorphic solutions of this generalised equation, when $n \geqslant 4$. Concerning the case $n=3$, we offer a counterexample to show that [7, Theorem 1] remains valid for $n \geqslant 4$ only.

We note that if $n=1$ then the equation (1.1) with meromorphic coefficients reduces into a linear differential equation, while if $n=2$ then (1.1) contains the first and the second Painlevé differential equations and the Riccati differential equations.

\section{Received 13th May, 2002}

This work was partially supported by the Academy of Finland, grant 50981, and by the INTAS project, grant 99-00089. The authors would like to thank Dr. J. Rieppo for valuable discussions.

Copyright Clearance Centre, Inc. Serial-fee code: 0004-9727/02 \$A2.00+0.00. 
In addition, we study the growth of meromorphic solutions of differential equations of the form

$$
L(f)+P(z, f)=h(z),
$$

where $L(f)$ has meromorphic coefficients, and $P(z, f)$ is a polynomial in $f$ with meromorphic coefficients.

\section{Preliminaries}

In what follows, a meromorphic function is always understood to be non-constant and meromorphic in the whole complex plane $\mathbb{C}$. Concerning the value distribution of meromorphic functions, we assume that the reader is familiar with basic notations such as $m(r, f), N(r, f), T(\dot{r}, f)$, and with basic results, see any standard reference like [2] or [4]. In particular, for a meromorphic function $f$, the notions of order

$$
\rho(f):=\limsup _{r \rightarrow \infty} \frac{\log ^{+} T(r, f)}{\log r},
$$

lower order

$$
\mu(f):=\liminf _{r \rightarrow \infty} \frac{\log ^{+} T(r, f)}{\log r},
$$

and type, when $0<\rho<\infty$,

$$
\tau(f):=\limsup _{r \rightarrow \infty} \frac{T(r, f)}{r^{\rho}},
$$

will appear frequently in the subsequent considerations. For the convenience of the reader, we recall the following Valiron-Mohon'ko theorem, see [5] or [4, pp. 31-34].

THEOREM A. Let $f$ be a meromorphic function. Then for all irreducible rational functions in $f$,

$$
R(z, f)=\frac{P(z, f)}{Q(z, f)}=\frac{\sum_{j=0}^{p} a_{j}(z) f^{j}}{\sum_{k=0}^{q} b_{k}(z) f^{k}}
$$

with meromorphic coefficients $a_{j}(z), b_{k}(z)$ such that

$$
\begin{cases}T\left(r, a_{j}\right)=S(r, f), & j=0, \ldots, p, \\ T\left(r, b_{k}\right)=S(r, f), & k=0, \ldots, q,\end{cases}
$$

the characteristic function of $R(z, f)$ satisfies

$$
T(r, R(z, f))=d T(r, f)+S(r, f)
$$

where $d=\max \{p, q\}$. 
REMARK. If we assume, in addition, that $a_{j}(z)$ and $b_{k}(z)$ are complex constants, then

$$
T(r, R(z, f))=d T(r, f)+O(1) .
$$

This follows by a careful examination of the proof of Theorem A.

Finally, we introduce two differential fields of meromorphic functions, needed throughout of this paper. Firstly, given a meromorphic function $f$, we define

$$
\mathcal{L}_{f}:=\{h \text { meromorphic }: T(r, h)=S(r, f)\} .
$$

Secondly, given $\rho>0$, we define

$$
\mathcal{L}_{\rho}:=\{h \text { meromorphic : } \rho(h)<\rho\} .
$$

\section{Growth CONSIDERATIONS}

In this section, we deal with differential equations of the form

$$
L(f)+P(z, f)=h(z)
$$

where $L(f)=a_{0}(z) f+a_{1}(z) f^{\prime}+\cdots+a_{k}(z) f^{(k)}$ is a linear differential polynomial in $f$ with meromorphic coefficients, $P(z, f)=b_{2}(z) f^{2}+\cdots+b_{n}(z) f^{n}$ is a polynomial in $f$ with meromorphic coefficients, and $h(z)$ is meromorphic. Note that the function $q_{0}(z)$ appeared in [7] is incorporated in $h(z)$. Throughout the paper, we denote $n:=\operatorname{deg}_{f} P(z, f)$. Moreover, we restrict ourselves to consider meromorphic solutions $f$ of (3.1) such that all coefficients of $L(f)$ and $P(z, f)$ are in $\mathcal{L}_{f}$. Provided $f$ has a few poles only, in the sense that $N(r, f)=S(r, f)$, it appears that all such solutions are of comparable growth. To see this, we prove the following.

TheOREM 3.1. Given $L(f), P(z, f), h(z)$ as to above, and $P(z, f) \not \equiv 0$, denote by $\mathcal{F}$ the family of meromorphic solutions of (3.1) such that whenever $f \in \mathcal{F}$, all coefficients of (3.1) are in $\mathcal{L}_{f}$, and $N(r, f)=S(r, f)$. If now $f, g \in \mathcal{F}$, then

$$
T(r, g)=O(T(r, f))+S(r, f) \text {. }
$$

Moreover, if $\alpha>1$, then for some $r_{\alpha}>0$,

$$
T(r, g)=O(T(\alpha r, f))
$$

for all $r \geqslant r_{\boldsymbol{\alpha}}$.

Proof: Since $f$ and $g$ are solutions of (3.1), we have

$$
\begin{aligned}
\frac{L(f)-L(g)}{f-g} & =-\frac{P(z, f)-P(z, g)}{f-g} \\
& =-\frac{b_{n}\left(f^{n}-g^{n}\right)+\cdots+b_{2}\left(f^{2}-g^{2}\right)}{f-g}
\end{aligned}
$$


for some $n \geqslant 2$. Hence, we may write

$$
\frac{L(f-g)}{f-g}=\frac{L(f)-L(g)}{f-g}=Q(z, f, g),
$$

where $Q(z, f, g)$ is a polynomial in $f$ and $g$ with coefficients in $\mathcal{L}_{f} \cap \mathcal{L}_{g}$ such that $\operatorname{deg}_{f} Q$ $=\operatorname{deg}_{g} Q \geqslant 1$. By the lemma of the logarithmic derivative,

$$
m(r, Q(z, f, g))=S(r, f)+S(r, g)
$$

Since

$$
\max \{N(r, f), N(r, g)\}=S(r, f)+S(r, g),
$$

it clearly follows that

$$
T(r, Q(z, f, g))=S(r, f)+S(r, g) \text {. }
$$

Now, (3.4) may be interpreted as an algebraic equation for $g$ over the field

$$
\mathcal{L}:=\{h \text { meromorphic }: T(r, h)=O(T(r, f))+S(r, f)+S(r, g)\} .
$$

Therefore, by a slight modification of [3, Theorem 1$]$,

$$
T(r, g)=O(T(r, f))+S(r, f)+S(r, g)
$$

from which (3.2) immediately follows, while (3.3) is a standard consequence of (3.2) by [4, Lemma 1.1.1].

We now proceed to consider meromorphic solutions $f$ of (3.1) such that all coefficients of (3.1) are in $\mathcal{L}_{f}$, giving no restrictions for possible poles of $f$, nor for the degree of $P(z, f)$. Note that in the case $P(z, f) \equiv 0$ the following result reduces to the well known fact in the theory of linear differential equations.

Proposition 3.2. If $f$ is a meromorphic solution of (3.1) such that all coefficients of $(3.1)$ are in $\mathcal{L}_{f}$, then $\rho(f) \geqslant \rho(h)$. Similarly for the lower order, $\mu(f) \geqslant \mu(h)$.

Proof: The equation (3.1) gives

$$
T(r, h) \leqslant T(r, L(f))+T(r, P(z, f))+O(1) .
$$

Since $a_{j} \in \mathcal{L}_{f}$ for all $j=1, \ldots, k$, we obtain

$$
T(r, L(f)) \leqslant(k+1) T(r, f)+S(r, f)
$$

by the Milloux estimate, see [2, p. 55]. Further, since $b_{j} \in \mathcal{L}_{f}$ for all $j=2, \ldots, n$, we obtain

$$
T(r, P(z, f))=n T(r, f)+S(r, f)
$$


by the Valiron-Mohon'ko theorem. Substituting (3.6) and (3.7) into (3.5) and applying [4, Lemma 1.1.1], we see that, for every $\varepsilon>0$ and $\alpha>1$, there exists an $R>0$ such that

$$
T(r, h) \leqslant(n+k+1+\varepsilon) T(\alpha r, f),
$$

provided $r \geqslant R$. Then

$$
\begin{aligned}
\rho(h) & \leqslant \limsup _{r \rightarrow \infty} \frac{\log T(\alpha r, f)+O(1)}{\log r} \\
& =\limsup _{r \rightarrow \infty} \frac{\log T(\alpha r, f)}{\log \alpha r-\log \alpha}=\rho(f) .
\end{aligned}
$$

The second inequality $\mu(h) \leqslant \mu(f)$ follows similarly from (3.8).

The following result offers a partial answer to the problem whether $\rho(f)=\rho(h)$ and $\mu(f)=\dot{\mu}(h)$ may appear in Proposition 3.2.

PROPOS ITION 3.3. Suppose $f$ is a meromorphic solution of (3.1) such that all coefficients of (3.1) are in $\mathcal{L}_{f}$. Denote $n:=\operatorname{deg}_{f} P(z, f) \geqslant 2$.

(a) If $n \geqslant k+2$, then $\rho(f)=\rho(h)$ and $\mu(f)=\mu(h)$.

(b) If $N(r, f)=S(r, f)$, then $\rho(f)=\rho(h)$ and $\mu(f)=\mu(h)$.

Proof: By Proposition 3.2, it suffices to show that $\rho(f) \leqslant \rho(h)$ and $\mu(f) \leqslant \mu(h)$.

(a) Write (3.1) in the form $P(z, f)=-L(f)+h(z)$. Then, using (3.6), (3.7) and [4, Lemma 1.1.1], it immediately follows that, for every $\varepsilon \in(0,1)$ and $\alpha>1$, there exists an $R>0$ such that

$$
(n-k-1-\varepsilon) T(r, f) \leqslant T(\alpha r, h),
$$

provided $r \geqslant R$. Since $n \geqslant k+2$ by the assumption, the left-hand side of (3.9) remains positive. Hence,

$$
\rho(f) \leqslant \limsup _{r \rightarrow \infty} \frac{T(\alpha r, h)}{\log (\alpha r)-\log \alpha}=\rho(h) .
$$

The identity $\mu(f)=\mu(h)$ follows in a similar way.

(b) By examining the proof of the Milloux estimate in $[2$, p. 55], we deduce that the assumption $N(r, f)=S(r, f)$ results in

$$
T(r, L(f)) \leqslant T(r, f)+S(r, f) .
$$

By making use of (3.10), we get, corresponding to (3.9), that

$$
(n-1-\varepsilon) T(r, f) \leqslant T(\alpha r, h),
$$

provided $r \geqslant R$. Since $n \geqslant 2$ by the assumption, the left-hand side of (3.11) remains positive. Exactly as in Part (a), we obtain Part (b).

EXAmples. We now list some examples related to Proposition 3.2 and 3.3, which show that all possible combinations between $\rho(f), \rho(h)$ and $\infty$ may appear: 
(a) $\infty>\rho(f)>\rho(h)$. We offer two examples in this case:

(i) The first Painleve differential equation is clearly of the form (3.1) with $\rho(f)=5 / 2(\operatorname{see}[6])$ and $h(z)=z$.

(ii) See [1, Example 13.4] for a Riccati differential equation with $h(z) \equiv 0$ possessing a meromorphic solution $f$ of order $q(2 m+1)$, where $q, m \in \mathbb{N}$ can be chosen arbitrarily.

(b) $\infty>\rho(f)=\rho(h)$. See Example 4.1 in the next section.

(c) $\infty=\rho(f)>\rho(h)$. For every $k \in \mathbb{N}$, function $f(z)=\exp (z)+\exp (\exp (z))$ satisfies

$$
f^{(k)}(z)-f(z) \sum_{j=1}^{k} e^{j z}=e^{z}-\sum_{j=2}^{k+1} e^{j z}
$$

(d) $\quad \infty=\rho(f)=\rho(h)$. For every $k \in \mathbb{N}$, function $f(z)=\exp (\exp (z))$ satisfies

$$
f^{(k)}(z)+f(z)\left(1-\sum_{j=1}^{k} e^{j z}\right)=e^{e^{z}}
$$

The inequalities (3.8), (3.9) and (3.11) lead us to the following statement concerning the types $\tau(f)$ and $\tau(h)$.

Proposition 3.4. Suppose $f$ is a meromorphic solution of (3.1) such that all coefficients of $(3.1)$ are in $\mathcal{L}_{f}$. Suppose further that $0<\rho(f)<\infty$. Denote $n:=\operatorname{deg}_{f} P(z, f) \geqslant 2$.

(a) If $n \geqslant k+2$, then

$$
(n-k-1) \tau(f) \leqslant \tau(h) \leqslant(n+k+1) \tau(f) .
$$

(b) If $N(r, f)=S(r, f)$, then

$$
(n-1) \tau(f) \leqslant \tau(h) \leqslant(n+1) \tau(f) .
$$

Proof: Using Proposition 3.3, we may denote $\rho=\rho(f)=\rho(h)$.

(a) Dividing (3.9) by $r^{\rho}$, we obtain

$$
(n-k-1-\varepsilon) \tau(f) \leqslant \limsup _{r \rightarrow \infty} \frac{T(\alpha r, h)}{r^{\rho}}=\alpha^{\rho} \tau(h) .
$$

Similarly, dividing (3.8) by $r^{\rho}$, we obtain

$$
\tau(h) \leqslant(n+k+1+\varepsilon) \limsup _{r \rightarrow \infty} \frac{T(\alpha r, f)}{r^{\rho}}=(n+k+1+\varepsilon) \alpha^{\rho} \tau(f) .
$$

By letting $\varepsilon \rightarrow 0$ and $\alpha \rightarrow 1$ in (3.12) and (3.13), we conclude the proof of Part (a). 
(b) Dividing (3.11) by $r^{\rho}$, we get

$$
(n-1-\varepsilon) \tau(f) \leqslant \limsup _{r \rightarrow \infty} \frac{T(\alpha r, h)}{r^{\rho}}=\alpha^{\rho} \tau(h) .
$$

By making use of (3.10), we get, corresponding to (3.8), that

$$
T(r, h) \leqslant(n+1+\varepsilon) T(\alpha r, f),
$$

provided $r \geqslant R$. Dividing (3.15) by $r^{\rho}$, we get

$$
\tau(h) \leqslant(n+1+\varepsilon) \limsup _{r \rightarrow \infty} \frac{T(\alpha r, f)}{r^{\rho}}=(n+1+\varepsilon) \alpha^{\rho} \tau(f) .
$$

Letting again $\varepsilon \rightarrow 0$ and $\alpha \rightarrow 1$ in (3.14) and (3.16), we conclude the proof of Part (b). 0

We close this section by giving a result on the ratio of the Nevanlinna characteristics of the functions $h$ and $f$. Note that there are no restrictions concerning $n, k$ and $\rho(f)$.

CoRollary 3.5. Suppose $f$ is a meromorphic solution of (3.1) such that all coefficients of $(3.1)$ are in $\mathcal{L}_{f}$. Let $\alpha>1$ be any fixed constant, and denote $n:=\operatorname{deg}_{f} P(z, f)$. Then

$$
\limsup _{r \rightarrow \infty} \frac{T(r, h)}{T(\alpha r, f)} \leqslant n+k+1
$$

and

$$
\liminf _{r \rightarrow \infty} \frac{T(\alpha r, h)}{T(r, f)} \geqslant n-k-1 .
$$

Suppose further that $N(r, f)=S(r, f)$ holds. Then

$$
\limsup _{r \rightarrow \infty} \frac{T(r, h)}{T(\alpha r, f)} \leqslant n+1
$$

and

$$
\liminf _{r \rightarrow \infty} \frac{T(\alpha r, h)}{T(r, f)} \geqslant n-1 .
$$

Proof: The first two inequalities follow from (3.8) and (3.9), respectively, while the remaining two inequalities follow from (3.15) and (3.11), respectively.

\section{MEROMORPHIC SOLUTIONS WITH FEW POLES}

This section is devoted to pointing out a slip in [7], and offering a slight improvement for [7] in the case $n \geqslant 4$. We start by giving an example which shows that [7, Theorem 1$]$ fails for $n=3$. 
EXAMPLE 4.1. Let $Q(z), p(z) \in \mathcal{L}_{1}=\{h: \rho(h)<1\}$, let $\alpha, q_{1}, q_{2} \in \mathbb{C} \backslash\{0\}$, and let

$$
P(z):=\frac{-Q(z)-p(z) \alpha^{2}}{q_{1} q_{2}} \not \equiv 0 .
$$

Moreover, suppose that $\sqrt{3}>0$, and denote

$$
h(z):=\frac{i \sqrt{3}}{9}\left(Q(z)+p(z) \alpha^{2}\right)\left(\frac{q_{2}^{2}}{q_{1}} e^{-3 \alpha z}-\frac{q_{1}^{2}}{q_{2}} e^{3 \alpha z}\right) .
$$

Then, assuming $\varepsilon_{1}=-(1 / 2)-(\sqrt{3} / 2) i$ and $\varepsilon_{2}=-(1 / 2)+(\sqrt{3} / 2) i$,

$$
f_{1}(z)=\frac{\varepsilon_{1} q_{2}-q_{1} e^{2 \alpha z} \varepsilon_{2}}{e^{\alpha z}\left(\varepsilon_{1}-\varepsilon_{2}\right)}
$$

and

are two solutions of

$$
f_{2}(z)=\frac{-q_{1} e^{2 \alpha z}+q_{2}}{e^{\alpha z}\left(\varepsilon_{1}-\varepsilon_{2}\right)}
$$

$$
p(z) f^{\prime \prime}+Q(z) f+P(z) f^{3}=h(z),
$$

such that $f_{1} \not \equiv \varepsilon_{j} f_{2}, j=1,2$.

By substituting $q_{1}=-(\sqrt{3} / 4)-(3 i / 4), q_{2}=-(\sqrt{3} / 4)+(3 i / 4), \alpha=i, Q(z) \equiv 0$ and $p(z) \equiv 3$, we see that the differential equation

$$
3 f^{\prime \prime}+4 f^{3}=-\sin (3 z)
$$

has at least two meromorphic solutions, namely $f_{1}(z)=\sin (z)$ and $f_{2}(z)=-(1 / 2) \sin z$ $-(1 / 2) \sqrt{3} \cos z$.

Observe that, for $k \geqslant 3$, (4.1) may be extended to

$$
f^{(k)}+f^{(k-2)}+3 f^{\prime \prime}+4 f^{3}=-\sin (3 z)
$$

which has the same two solutions $f_{1}$ and $f_{2}$ as (4.1).

Restricting ourselves first to the case $n \geqslant 4$, we may apply the ideas from the proof of [7, Theorem 1], to obtain the following slight extension of [7]:

THEOREM 4.2. Let $f$ be a meromorphic function such that $N(r, f)=S(r, f)$. Moreover, let $n, k$ be positive integers, with $n \geqslant 4$, let $p(z) \in \mathcal{L}_{f}, p$. $\not \equiv 0$, and let $L(f)$ denote a linear differential polynomial in $f$,

$$
L(f)=a_{0}(z) f+a_{1}(z) f^{\prime}+\cdots+a_{k}(z) f^{(k)},
$$

where $a_{j}(z) \in \mathcal{L}_{f}$ for all $j=0, \ldots, k$ such that not all $a_{j}$ are identically zero. Moreover, let $h(z)$ be a meromorphic function. If $f$ is a solution of the nonlinear differential equation

$$
L(f)-p(z) f^{n}=h(z)
$$

then one of the following situations hold: 
(a) The equation (4.2) has $f$ as its unique transcendental meromorphic solution such that $N(r, f)=S(r, f)$.

(b) The equation (4.2) has exactly $n$ transcendental meromorphic solutions $f_{j}$, $j=1, \ldots, n$, such that $N\left(r, f_{j}\right)=S\left(r, f_{j}\right)$. In this case, $L\left(f_{j}\right) \equiv 0$ and $h(z)=-p(z) f_{j}^{n}$ for all $j=1, \ldots, n$.

(c) The equation (4.2) has $f$ as its unique polynomial solution.

(d) The equation (4.2) has exactly $n$ polynomial solutions $f_{j}, j=1, \ldots, n$. In this case, $L\left(f_{j}\right) \equiv 0$ and $h(z)=-p f_{j}^{n}, p \in \mathbb{C}$, for all $j=1, \ldots, n$.

Proof: Suppose that (4.2) has at least two distinct transcendental meromorphic solutions $f, g$ such that $N(r, f)=S(r, f)$ and $N(r, g)=S(r, g)$. Then,

$$
L(f)-L(g)-p(z)\left(f^{n}-g^{n}\right)=0,
$$

and so

$$
\frac{L(f-g)}{f-g}=p(z) \frac{f^{n}-g^{n}}{f-g}=p(z) F(z),
$$

where $F(z)=\left(f-\varepsilon_{1} g\right)\left(f-\varepsilon_{2} g\right) \cdots\left(f-\varepsilon_{n-1} g\right)$. Here $\varepsilon_{j} \neq 1$ are the distinct $n$ :th roots of unity. Now,

$$
m(r, F)=m\left(r, \frac{1}{p} \cdot \frac{L(f)-L(g)}{f-g}\right)=S(r, f-g) \leqslant S(r, f)+S(r, g)
$$

and $N(r, F)=S(r, f)+S(r, g)$ by assumption. Hence $T(r, F)=S(r, f)+S(r, g)$.

Assume first that $F \equiv 0$. Then $f^{n}=g^{n}$, and $g=\varepsilon_{j} f$ for some $j=1, \ldots, n-1$. By substituting this into (4.3), we have that $L(f)-L\left(\varepsilon_{j} f\right)=\left(1-\varepsilon_{j}\right) L(f)=0$, and so $L(f)=0$. Thus $h(z)=-p(z) f^{n}$ as asserted. Moreover, $L\left(\varepsilon_{j} f\right)=0$ for all $j=1, \ldots, n-1$. Therefore, $f, \varepsilon_{1} f, \ldots, \varepsilon_{n-1} f$ are the solutions of (4.2), completing the proof of the Part (b).

Assume now that $F \not \equiv 0$. Then

$$
\begin{aligned}
F(z) & =f^{n-1}\left(1-\varepsilon_{1} \frac{g}{f}\right)\left(1-\varepsilon_{2} \frac{g}{f}\right) \cdots\left(1-\varepsilon_{n-1} \frac{g}{f}\right) \\
& =\varepsilon_{1} \varepsilon_{2} \cdots \varepsilon_{n-1} f^{n-1}\left(\varepsilon_{1}-\frac{g}{f}\right)\left(\varepsilon_{2}-\frac{g}{f}\right) \cdots\left(\varepsilon_{n-1}-\frac{g}{f}\right) \\
& = \pm f^{n-1}\left(\varepsilon_{1}-\frac{g}{f}\right)\left(\varepsilon_{2}-\frac{g}{f}\right) \cdots\left(\varepsilon_{n-1}-\frac{g}{f}\right) .
\end{aligned}
$$

Now, if $(g / f)\left(z_{0}\right)=\varepsilon_{j}$ for some $j=1, \ldots, n-1$, then either $F\left(z_{0}\right)=0$, or $f\left(z_{0}\right)=\infty$. Since $T(r, F)=S(r, f)+S(r, g)$ and $N(r, f)=S(r, f)$, it follows that

$$
\bar{N}\left(r, \frac{1}{(g / f)-\varepsilon_{j}}\right)=S(r, f)+S(r, g)
$$

for all $j=1, \ldots, n-1$. We have by (4.4) that

$$
\frac{F(z)}{f^{n-1}}=Q\left(\frac{g}{f}\right)
$$


where $Q$ is a polynomial in $g / f$ of degree $n-1$ with constant coefficients. By Remark following Theorem $\mathrm{A}$,

$$
(n-1) T\left(r, \frac{g}{f}\right)+O(1)=T\left(r, \frac{F}{f^{n-1}}\right)=(n-1) T(r, f)+S(r, f)+S(r, g) .
$$

On the other hand,

$$
\frac{F(z)}{g^{n-1}}= \pm Q\left(\frac{f}{g}\right)
$$

where $Q$ is the same polynomial as to above. Again by Theorem A,

$$
\begin{aligned}
(n-1) T\left(r, \frac{g}{f}\right)+O(1) & =(n-1) T\left(r, \frac{f}{g}\right)+O(1)=T\left(r, \frac{F}{g^{n-1}}\right) \\
& =(n-1) T(r, g)+S(r, f)+S(r, g) .
\end{aligned}
$$

Combining (4.6) and (4.7), we obtain

$$
T(r, f)+S(r, f)=T(r, g)+S(r, g) .
$$

Hence $S(r, g)=S(r, f)$, and so by (4.6),

$$
T\left(r, \frac{g}{f}\right)=T(r, f)+S(r, f) .
$$

Thus $S(r, f)=S(r,(g / f))$, and, by the second main theorem [4, p. 48] and (4.5), we obtain

$$
(n-3) T\left(r, \frac{g}{f}\right) \leqslant \sum_{j=1}^{n-1} \bar{N}\left(r, \frac{1}{(g / f)-\varepsilon_{j}}\right)=S(r, f)=S\left(r, \frac{g}{f}\right) .
$$

This is a contradiction, since we assumed $n \geqslant 4$. Therefore equation (4.2) may have only one transcendental meromorphic solution such that $N(r, f)=S(r, f)$, as asserted in Part (a).

Suppose now that (4.2) has a polynomial solution $f$ satisfying the assumptions of the theorem. Then the coefficients $p, a_{0}, \ldots, a_{k}$ must be constants. By Theorem 3.1, if (4.2) has another meromorphic solution $g$ satisfying the assumptions of Theorem 4.2, then $g$ has to be rational and, in fact, a polynomial, since $N(r, g)=S(r, g)$. Therefore, we may now assume that (4.2) has at least two distinct polynomial solutions $f, g$.

Exactly as in the first part of the proof,

$$
\frac{L(f-g)}{f-g}=p F(z)=p\left(f-\varepsilon_{1} g\right) \cdots\left(f-\varepsilon_{n-1} g\right) .
$$

Moreover, since $f$ and $g$ are polynomials,

$$
T(r, F)=m(r, F)=m\left(r, \frac{1}{p} \frac{L(f-g)}{f-g}\right)=O(1)
$$


and so $F$ has to be a constant. If $F \equiv 0$, the same reasoning as for Part (b) results in the situation of Part (d). To proceed, assume that $F \not \equiv 0$. Since by (4.8)

$$
\operatorname{deg}_{z} F=\sum_{j=1}^{n-1} \operatorname{deg}_{z}\left(f-\varepsilon_{j} g\right)=0
$$

we conclude that $f-\varepsilon_{j} g$ is a constant, for $j=1, \ldots, n-1$. Hence $f$ and $g$ must be constants, a contradiction. Therefore, the equation (4.2) may have only one polynomial solution, as asserted in Part (c).

EXAMPLES. The following list of examples demonstrates that each of the cases (a)-(d) may occur in Theorem 4.2 .

(a) Suppose that $f$ is a meromorphic solution of the differential equation

$$
10 f^{\prime \prime}+16 f^{5}=\sin (5 z)-5 \sin (3 z) .
$$

We immediately see, by standard pole multiplicity consideration, that $f$ must be entire. Therefore by Theorem $4.2, f(z)=\sin (z)$ is the unique meromorphic, in fact entire, solution of (4.9).

(b) The equation $f^{\prime \prime}+f+f^{4}=(\sin z)^{4}$ has exactly four transcendental meromorphic solutions $f_{j}(z)=\varepsilon_{j} \sin (z)$.

(c) The equation $f^{\prime}+f^{4}=z^{4}+1$ has exactly one polynomial solution $f(z)=z$.

(d) The equation $f^{\prime \prime}+f^{4}=z^{4}$ has exactly four polynomial solutions $f_{j}(z)$ $=\varepsilon_{j} z$.

Returning back to the case $n=3$ in Theorem 4.2, the proof of Theorem 4.2 shows that if (4.2) has a polynomial solution, then either (c) or (d) holds, including the case $n=3$. On the other hand, Example 4.1 demonstrates that neither (a) nor (b) need to be valid for transcendental meromorphic solutions in the case $n=3$ and $k \geqslant 2$ arbitrary. We now proceed to show that (a) and (b) of Theorem 4.2 remain valid for $n=3$, provided $k=1$.

THEOREM 4.3. Let $f$ be a transcendental meromorphic function, suppose $n=3$ and $k=1$ and suppose that all the other assumptions in Theorem 4.2 hold. If $f$ satisfies the nonlinear differential equation

$$
a_{1}(z) f^{\prime}+a_{0}(z) f-p(z) f^{3}=h(z)
$$

then one of the following situations hold:

(a) The equation (4.10) has $f$ as its unique transcendental meromorphic solution such that $N(r, f)=S(r, f)$. 
(b) The equation (4.10) has exactly three transcendental meromorphic solutions $f_{j}, j=1,2,3$ such that $N\left(r, f_{j}\right)=S\left(r, f_{j}\right)$ for $j=1,2,3$. Moreover $a_{1}(z) f_{j}^{\prime}+a_{0}(z) f_{j} \equiv 0$, and $h(z)=-p(z) f_{j}^{3}$ for all $j=1,2,3$.

PROOF: Suppose that (4.2) has at least two distinct transcendental meromorphic solutions $f, g$ such that $N(r, f)=S(r, f)$ and $N(r, g)=S(r, g)$. Then, similarly as in the proof of Theorem 4.2,

$$
\frac{a_{1}(z)\left(f^{\prime}-g^{\prime}\right)+a_{0}(z)(f-g)}{f-g}=p(z) \frac{f^{3}-g^{3}}{f-g}=p(z) F(z),
$$

where $F(z)=\left(f-\varepsilon_{1} g\right)\left(f-\varepsilon_{2} g\right)$. Here $\varepsilon_{j} \neq 1$ are the distinct third roots of unity. Now,

$$
\begin{aligned}
m(r, F) & =m\left(r, \frac{1}{p} \cdot \frac{a_{1}\left(f^{\prime}-g^{\prime}\right)+a_{0}(f-g)}{f-g}\right) \\
& =S(r, f-g) \leqslant S(r, f)+S(r, g)
\end{aligned}
$$

and

$$
N(r, F)=S(r, f)+S(r, g)
$$

by assumption. Again, by repeating the reasoning in the proof of Theorem 4.2, we have that $S(r, g)=S(r, f)$ and $T(r, F)=S(r, f)+S(r, g)=S(r, g / f)$. The case $F \equiv 0$ leads to the assertion of Part (b) exactly as in the proof of Theorem 4.2, and so we may assume that $F \not \equiv 0$. Then,

$$
\bar{N}\left(r, \frac{1}{(g / f)-\varepsilon_{j}}\right)=S\left(r, \frac{g}{f}\right), \quad j=1,2 .
$$

Assume now that $z_{0} \in \mathbb{C}$ is such that $(g / f)\left(z_{0}\right)=1$. Then $(f-g)\left(z_{0}\right)=0$, which is possible only when either $F$ has a pole at $z_{0}$, or one of the coefficient functions of (4.11) has a pole or zero at $z_{0}$. But this means that

$$
\bar{N}\left(r, \frac{1}{(g / f)-1}\right)=S\left(r, \frac{g}{f}\right)
$$

and so, by (4.12) and by the second main theorem,

$$
T\left(r, \frac{g}{f}\right) \leqslant \sum_{j=0}^{2} \bar{N}\left(r, \frac{1}{(g / f)-\varepsilon_{j}}\right)=S\left(r, \frac{g}{f}\right),
$$

where $\varepsilon_{0}:=1$. This is a contradiction. Therefore equation (4.10) may have only one transcendental meromorphic solution such that $N(r, f)=S(r, f)$, as asserted in Part (a). 


\section{REFERENCES}

[1] G.G. Gundersen and I. Laine, 'On the meromorphic solutions of some algebraic differential equations', J. Math. Anal. Appl. 111 (1985), 281-300.

[2] W.K. Hayman, Meromorphic functions (Clarendon Press, Oxford, 1964).

[3] S. Hellerstein and L.A. Rubel, 'Subfields that are algebraically closed in the field of meromorphic functions', J. Anal. Math. 12 (1964), 105-111.

[4] I. Laine, Nevanlinna theory and complex differential equations (Walter de Gruyter, Berlin, 1993).

[5] A.Z. Mohon'ko, 'The Nevanlinna characteristic of certain meromorphic functions', (Russian), Teor. Funktsii Funktsional. Anal. i Prilozhen 14 (1971), 83-87.

[6] N. Steinmetz, 'Value distribution of the Painlevé transcendents', Israel J. Math. 128 (2002), 29-52.

[7] C.-C. Yang, 'On entire solutions of a certain type of nonlinear differential equation', Bull. Austral. Math. Soc. 64 (2001), 377-380.

Department of Mathematics

University of Joensuu

P.O. Box 111

FIN-80101 Joensuu

Finland

e-mail: heittoka@cc.joensuu.fi

rkorhone@cc.joensuu.fi

ilpo.laine@joensuu.fi 